\title{
Accessory head of flexor pollicis longus in Jeju islander cadavers
}

\author{
By Jae Ma Yu ${ }^{1}$, Sang Pil Yoon ${ }^{2}$, Jinu Kim²,* \\ ${ }^{1}$ Medical Course, Jeju National University School of Medicine, Jeju 63243, Republic of Korea \\ ${ }^{2}$ Department of Anatomy, Jeju National University School of Medicine, Jeju 63243, Republic of Korea
}

\begin{abstract}
Anterior interosseous nerve palsy is known to occur uncommonly because of its compression by the accessory head of flexor pollicis longus (AHFPL) in the forearm. During routine educational dissection, we found 7 AHFPLs in 12 upper limbs of 6 adults Korean Jeju islander cadavers, which inserted onto flexor pollicis longus. Three AHFPLs of them arose from coronoid process of the ulna, and the others arose independently from the flexor digitorum superficialis (FDS). Using the topographical relationship of the anterior interosseous nerve to the AHFPL, all anterior interosseous nerve was crossed the tendinous part of the AHFPL. This study has shown that there are discrepancies in the origin of AHFPL and the location of the anterior interosseous nerve in Koreans, which is supposed to be related to unique genetic pool in Jeju Island.
\end{abstract}

Key words: Accessory head of flexor pollicis longus, Anterior interosseous nerve

\section{INTRODUCTION}

In the deep layer of the forearm, flexor pollicis longus (FPL) located laterally to flexor digitorum profundus (FDP) arises from the grooved anterior surface of the radius and the adjacent interosseous membrane, and inserts onto the base of the distal phalanx of the thumb. Anterior interosseous nerve which comes from the median nerve in the elbow innervates FPL as well as pronator quadratus and the radial part of FDP in the forearm. In 1813, the accessory head of the flexor pollicis longus (AHFPL) was first described by CFL Gantzer, ${ }^{1)}$ since then it has been subsequently studied by a number of researchers. ${ }^{2-8)}$ AHFPL contributes to anterior interosseous nerve palsy through the compression of the nerve. The patient who has anterior interosseous neuropathy cannot make an "okay sign" with the thumb and forefinger. Although well known to anatomists and physicians, the Korean, especially Jeju islander, AHFPL has rarely been evaluated. In

Received: April 24, 2018; Revised: May 30, 2018; Accepted: May 31, 2018

*Correspondence to : Jinu Kim, Ph.D.

Department of Anatomy, Jeju National University School of Medicine, 102

Jejudaehak-ro, Jeju 63243, Republic of Korea

Tel: 82-64-754-8181, Fax: 82-64-702-2687

E-mail: jinu.kim@jejunu.ac.kr the present study, we found 7 AHFPLs in 12 upper limbs of 6 adults Korean cadavers.

\section{CASE REPORTS}

Twelve upper limbs of 6 adults Korean cadavers lived in Jeju Island (5 male and 1 female) were educationally dissected in Jeju National University School of Medicine. During that, we have found AHFPL and conducted to check its morphology.

As shown in Table 1, the AHFPL was found in 7 of 12 upper limbs. It appeared bilaterally in 3 and unilaterally in only 1 (right side) of the 6 individuals. Furthermore,

Table 1. Origin and insertion of AHFPL

\begin{tabular}{lcccccc}
\hline \multirow{2}{*}{$\begin{array}{c}\text { Cadaver } \\
\text { no. }\end{array}$} & \multicolumn{2}{c}{ Left forearm } & & \multicolumn{2}{c}{ Right forearm } \\
\cline { 2 - 3 } \cline { 5 - 6 } (gender) & Origin & Insertion & & Origin & Insertion \\
\hline 1 (male) & - & - & & - & - \\
2 (female) & - & - & & FDS & FPL \\
3 (male) & FDS & FPL & & FDS & FPL \\
4 (male) & FDS & FPL & Coronoid process & FPL \\
5 (male) & Coronoid process & FPL & & Coronoid process & FPL \\
6 (male) & - & - & & - & - \\
\hline FDS, flexor digitorum superficialis; & FPL, flexor pollicis longus; - , non-AHFPL
\end{tabular}


a

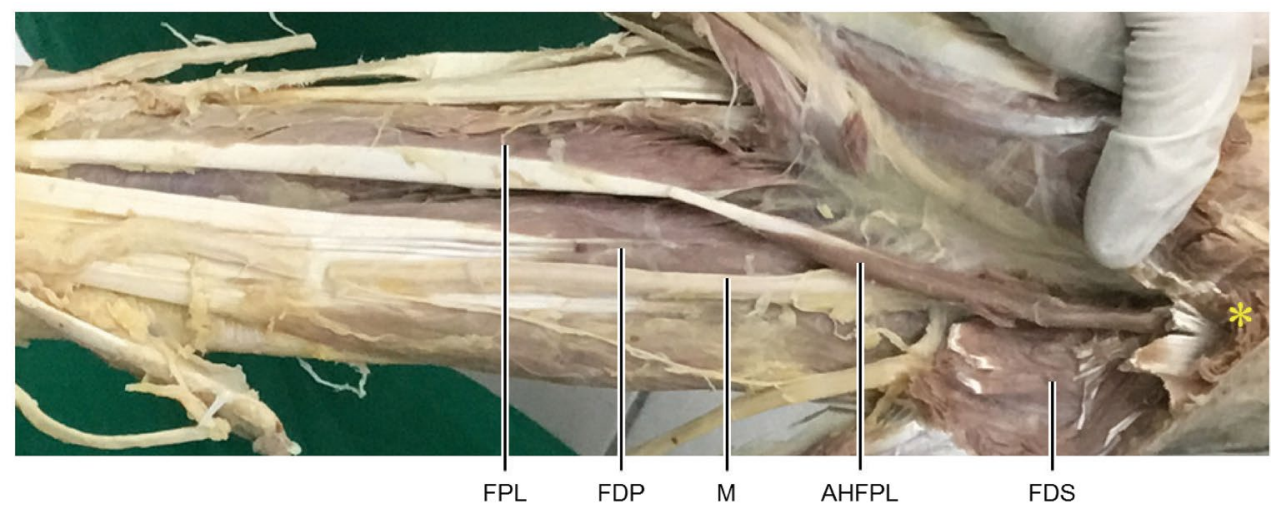

b

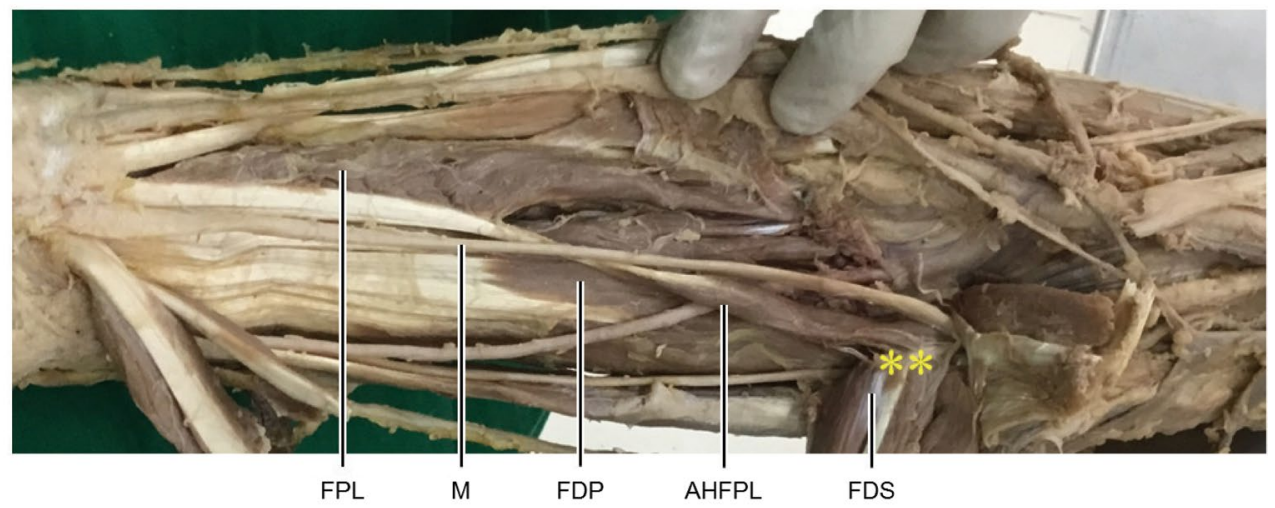

Figure 1. The accessory head of the flexor pollicis longus (AHFPL) originating from the coronoid process $(*)$ of the ulna (a) and the flexor digitorum superficialis (FDS, **) (b). FPL, flexor pollicis longus; M, median nerve; FDP, flexor digitorum profundus; FDS, flexor digitorum superficialis.

Table 2. Three types based on the topographical relationship to the anterior interosseous nerve to AHFPL.

\begin{tabular}{cl}
\hline Type & Topographical relationship \\
\hline A & Anterior interosseous nerve crosses the muscular part of AHFDL \\
B & Anterior interosseous nerve crosses the tendinous part of AHFDL \\
C & Anterior interosseous nerve coursed lateral to the AHFPL \\
\hline
\end{tabular}

two distinct types of its origin were revealed. It arose from either coronoid process of the ulna (3 of 7 AHFPLs) or independently from the flexor digitorum superficialis (FDS) (4 of 7 AHFPLs) (Fig. 1). All AHFPL inserted onto FPL.

As shown in Table 2, the topographical relationship of the anterior interosseous nerve to the AHFPL could be classified into $\mathrm{A}, \mathrm{B}$, and $\mathrm{C}$ types in dependence on the position at which the anterior interosseous nerve crossed the AHFPL before passing deep to the FPL: A type, the nerve crossed the muscular part of the AHFPL; B type, the nerve crossed the tendinous part of the AHFPL; and $\mathrm{C}$ type, it coursed lateral to the AHFPL. ${ }^{9)}$ In the present study, all anterior interosseous nerve passed under the tendon of AHFPL and FPL was classified into B type.

\section{DISCUSSION}

The incidence of the AHFPL is quite high, as $45.0 \%$ in American provide by Dellon, ${ }^{6}$ 52.0\% in Saudi Arabian provided by al-Qattan, $54.2 \%$ in Indian provided by Malhotra, ${ }^{4)}$ and $62.1 \%$ in Thailandian provided by Mahakkanukrauh, ${ }^{3)}$ and $66.7 \%$ in Korean provided by Oh. ${ }^{7)}$ Its incidence was revealed more commonly in men than in women, and more frequent on the right forearm than on the left forearm. Consistent with previous studies, ${ }^{3-8)}$ the overall incidence of the AHFPL obtained in this study was high and more frequent on the right forearm than on the left forearm.

In the previous result reported by Roy, ${ }^{10)}$ the most common origin of the AHFPL was the medial epicondyle of humerus (43.6\%), which is followed by orderly many areas including coronoid process of ulna $(25.8 \%)$, dual 
origins from medial epicondyle and coronoid process $(16.1 \%)$, FDS $(0.7 \%)$, and muscle fascia $(0.2 \%)$. The previous report also showed that it was commonly inserted into FPL (94.6\%) followed by FDP (5.4\%). However, our results have shown that the most common origin of the AHFPL is the FDS (4 per 7 AHFPLs) followed by the coronoid process of ulna ( 3 per 7 AHFPLs). Furthermore, all AHFPL insertion is the FPL(7 per 7 AHFPLs).

Anterior interosseous nerve palsy is characterized by weakness in flexion of the interphalangeal joint of the thumb and the distal interphalangeal joints of the index and middle fingers, together with weakness of pronation when the elbow is flexed. ${ }^{11)}$ The cause of its palsy is implicated in the unusual compression of the nerve by the AHFPL. ${ }^{12)}$ Using the topographical relationship of the anterior interosseous nerve to the AHFPL, the previous study has reported that A type is observed most frequently (56.3\%), followed by C type (33.3\%), and B type $(10.4 \%) .{ }^{9)}$ However, in our AHFPLs, all anterior interosseous nerve was B type. Because B type is located between two muscle bellies, the anterior interosseous nerve could be less compressed by either FPL or FDP rather than $A$ and $C$ type; suggesting that $B$ type might be prone to be less possible to occur the anterior interosseous nerve neuropathy.

In conclusion, although our study was limited by 6 cadavers, there are discrepancies in the origin of AHFPL and the location of the anterior interosseous nerve in Koreans, which is supposed to be related to unique genetic pool in Jeju Island.

\section{REFERENCES}

1. Gantzer C. Dissertatio anatomica musculorum varietates sistens. Joannis Friderici, Berlin; 1813.
2. Pai MM, Nayak SR, Krishnamurthy A, Vadgaonkar R, Prabhu LV, Ranade AV, et al. The accessory heads of flexor pollicis longus and flexor digitorum profundus: incidence and morphology. Clin Anat 2008;21:252-8.

3. Mahakkanukrauh P, Surin P, Ongkana N, Sethadavit M, Vaidhayakarn P. Prevalence of accessory head of flexor pollicis longus muscle and its relation to anterior interosseous nerve in Thai population. Clin Anat 2004;17:631-5.

4. Malhotra V, Sing N, Tewari S. The accessory head of the flexor pollicis longus muscle and its nerve supply. Anat Anz 1982; 151:503-5.

5. Al-Qattan M. Gantzer's muscle: an anatomical study of the accessory head of the flexor pollicis longus muscle. J Hand Surg Br 1996;21:269-70.

6. Dellon A, Mackinnon SE. Musculoaponeurotic variations along the course of the median nerve in the proximal forearm. J Hand Surg Br 1987;12:359-63.

7. Oh CS, Chung IH, Koh KS. Anatomical study of the accessory head of the flexor pollicis longus and the anterior interosseous nerve in Asians. Clin Anat 2000;13:434-8.

8. Lee S-W, Lee J-H, Lee H. Double Gantzer's Muscles by Four Muscle Bellies and Its Clinical Significance: A Case Report. Korean J Phys Anthropol 2017;30:67-70.

9. Oh CS, Chung IH, Koh KS. Anatomical study of the accessory head of the flexor pollicis longus and the anterior interosseous nerve in Asians. Clin Anat 2000;13:434-8.

10. Roy J, Henry BM, Pekala PA, Vikse J, Ramakrishnan PK, Walocha JA, et al. The prevalence and anatomical characteristics of the accessory head of the flexor pollicis longus muscle: a meta-analysis. PeerJ 2015;3:e1255.

11. Spinner M. The anterior interosseous nerve syndrome. J Bone Joint Surg [Am] 1970;52:84-94.

12. Gardner-Thorpe C. Anterior interosseous nerve palsy: spontaneous recovery in two patients. J Neurol Neurosurg Psychiatry 1974;37:1146-50. 